\title{
Effect of proctocolectomy on serum antineutrophil cytoplasmic antibodies in patients with chronic ulcerative colitis
}

Tampere University Hospital, Tampere, Finland:

Department of

Surgery

P Aitola

M Matikainen

\section{Department of} Clinical Microbiology

A Miettinen

A Mattila

E Soppi

Correspondence to: Dr Petri Aitola, Department of Surgery, Tampere University Hospital,

PO Box 2000, FIN-33521 Tampere, Finland.

Accepted for publication 1 December 1994

\begin{abstract}
Aims-To study the effect of proctocolectomy on the antineutrophil cytoplasmic antibody (ANCA) titres in association with ulcerative colitis. Methods-Serum samples were taken from 15 patients with ulcerative colitis immediately before and at a mean of 24 months after proctocolectomy. Indirect immunofluorescence for ANCA and enzyme immunoassays for myeloperoxidase and proteinase-3 antibodies were employed. A liver biopsy was taken from every patient during the proctocolectomy, and serum liver enzyme activities were also determined.
\end{abstract}

Results-Before proctocolectomy, 13 of the 15 patients had perinuclear antineutrophil cytoplasmic antibodies (pANCA). Additionally, one patient had a low titre of classical cytoplasmic ANCA and one had granulocyte specific antinuclear antibodies. After proctocolectomy, the ANCA titres decreased in 10 patients, in two of whom they became negative. The titres remained the same in four patients with positive ANCA and increased twofold in one patient. Only one patient was proteinase- 3 antibody positive and all 15 patients were myeloperoxidase antibody negative. The clinical condition improved in all patients, irrespective of the ANCA status after proctocolectomy. Seven patients, all of whom were positive for $p$ ANCA before proctocolectomy, had histological liver abnormalities. No correlation was observed between serum liver enzyme levels and ANCA staining patterns or titres.

Conclusions-Proctocolectomy decreased the ANCA titres in the majority of our patients, suggesting that reduction of the inflammation or the available antigenic material modifies the immune disturbance related to ulcerative colitis.

(F Clin Pathol 1995;48:645-647)

Keywords: Antineutrophil cytoplasmic antibodies, proctocolectomy, liver, ulcerative colitis.

An association with antineutrophil cytoplasmic antibodies (ANCA) has been established in a variety of primary vasculitic syndromes ${ }^{1}$ and more recently with inflammatory bowel disease. ${ }^{23}$ Perinuclear antineutrophil cytoplasmic antibodies ( $\mathrm{p}$-ANCA), the predominant immunofluorescence pattern in inflam- matory bowel disease, have been detected in up to $84 \%$ of sera of patients with ulcerative colitis, differentiating it from Crohn's disease and other colitides. ${ }^{23}$ In contrast to vasculitides, especially Wegener's granulomatosis, where a correlation between disease activity and ANCA has been shown, ${ }^{4}$ most studies have not found a correlation between ANCA and the clinical activity or extent of ulcerative colitis. ${ }^{25-7}$ Whether ANCA in ulcerative colitis is merely an epiphenomenon related to colonic mucosal inflammation or reflects disturbances in immune regulation is still unclear.

Patients with ulcerative colitis may have extraintestinal manifestations, of which liver abnormalities are the most frequent. Primary sclerosing cholangitis has been shown to occur in $3.7 \%$ of patients with ulcerative colitis, ${ }^{8}$ and about $70 \%$ of patients with primary sclerosing cholangitis have concomitant ulcerative colitis. The aetiology and pathogenesis of these two conditions are still unknown, although autoimmune mechanisms are suspected. The detection of p-ANCA in over $80 \%$ of both diseases suggests a shared immunopathogenic mechanism between these diseases. ${ }^{79}$

Proctocolectomy has been successfully used in the surgical treatment of ulcerative colitis. However, reports on the effect of proctocolectomy on ANCA in ulcerative colitis are controversial. Positive p-ANCA has also been detected in ulcerative colitis patients after colectomy. ${ }^{236910}$

In the present study we investigated the effect of proctocolectomy on the ANCA titres in patients with ulcerative colitis. In view of the close relationship of ulcerative colitis and liver abnormalities, the association of ANCA with liver biopsy findings in these patients was also addressed.

\section{Methods}

The study population consisted of 15 patients (four females, 11 males; mean age 33 years, range 23 to 46 years) subjected to proctocolectomy because of ulcerative colitis. Thirteen patients had been treated with corticosteroids continuously or intermittently and two with sulphasalazine before the operation. Thirteen patients had total and two had left sided ulcerative colitis. The mean duration of the disease before proctocolectomy was $7 \cdot 3$ years (range 1 to 28 years). The indication for surgery was chronic active disease in 13 patients and dysplasia of the colonic mucosa in two. Fourteen patients had ileonal anastomosis and one had a conventional ileostomy performed. Serum samples were taken immediately before and at a median of 23 months (range 11 to 62 
Different immunofluorescence patterns of serum antineutrophil cytoplasmic antibodies $(A N C A)$ observed in 15 patients with ulcerative colitis before and after proctocolectomy.

\begin{tabular}{lcc}
\hline ANCA staining pattern & Before proctocolectomy & After proctocolectomy \\
\hline c-ANCA & $1(7 \%)$ & $1(7 \%)$ \\
p-ANCA & $13(86 \%)$ & $11(73 \%)$ \\
GS-ANA & $1(7 \%)$ & $1(7 \%)$ \\
Negative & 0 & $2(13 \%)$ \\
\hline
\end{tabular}

c-ANCA = classical cytoplasmic ANCA; -ANCA = perinuclear ANCA; GS-ANA = granulocyte specific antinuclear antibodies.

\section{Results}

The different ANCA immunofluorescence patterns observed before and after proctocolectomy are shown in the table. All 15 patients were positive for ANCA before and 13 (87\%) after proctocolectomy. Thirteen patients $(87 \%)$ had p-ANCA before proctocolectomy. One patient was positive for c-ANCA and one for GS-ANA before proctocolectomy. One patient tested positive for proteinase- 3 antibodies by enzyme immunoassay preoperatively and showed a sixfold decrease in levels of this antibody after proctocolectomy, associated with a simultaneous decrease of the p-ANCA titre from 200 to negative. He showed no symptoms or signs of systemic vasculitis at the time of operation and the clinical condition after one year's follow up was normal. None of the patients was myeloperoxidase antibody positive before or after proctocolectomy.

The changes in ANCA titres after proctocolectomy are shown in the figure. In nine of the 13 patients positive for p-ANCA, the titres decreased; in two of these they turned negative. In three patients the titres remained the same. In one patient only a twofold higher p-ANCA titre was observed 22 months after proctocolectomy. The mean p-ANCA titres were significantly lower after proctocolectomy than before it $(p<0.0069)$. Also in the patient with positive GS-ANA before proctocolectomy the titre decreased after the operation. In the patient with a low titre of c-ANCA preoperatively the titre remained the same after proctocolectomy. The clinical condition improved in all patients, irrespective of the ANCA status after proctocolectomy.

Six patients had normal histology at liver biopsy, two had mild steatosis, four had nonspecific reactive hepatitis, and three had fibrous non-suppurative cholangitis (one stage I, one stage II with primary sclerosing cholangitis proven by cholangiography, and one stage III). All patients with fibrous cholangitis or nonspecific reactive hepatitis at liver biopsy were positive for p-ANCA. No significant difference in ANCA titres was observed between patients with different liver biopsy findings. No correlation was found between ANCA titres and serum liver enzyme levels (data not shown). Follow up liver enzyme values showed no signs of worsening of the liver function in any of the patients after proctocolectomy.

\section{Discussion}

The three main staining patterns of ANCA, namely c-ANCA, p-ANCA, and GS-ANA, are detected with different disease associations. ${ }^{16}$ The predominant staining pattern in ulcerative colitis and primary sclerosing cholangitis has been shown to be atypical p-ANCA, which has been found in up to $84 \%$ of patients with ulcerative colitis. ${ }^{2}$ So far, there are only a few reports on the effect of proctocolectomy on p-ANCA in ulcerative colitis. ${ }^{269}$ Duerr et $a l^{\beta}$ reported a group of 27 postcolectomy ulcerative colitis patients without statistically lower levels of ANCA than in a different group of 40 ulcerative colitis patients without prior surgery. Reumaux et $a l^{10}$ showed no statistical difference in the presence of p-ANCA IN $49 \%$ of 70 ulcerative colitis patients not operated on, as
The change in antineutrophil cytoplasmic (ANCA) titres in 15 patients with ulcerative colitis before and after proctocolectomy. p-ANCA = perinuclear $A N C A$; $c-A N C A=$ classical cytoplasmic ANCA; GS-ANA $=$ granulocyte specific antinuclear antibody. 
compared to $34 \%$ of 32 ulcerative colitis patients with prior proctocolectomy. In contrast, Rump et $a l^{17}$ have suggested that a correlation exists between disease activity and pANCA titre in ulcerative colitis. Furthermore, they have reported two patients with ulcerative colitis who became negative for ANCA after proctocolectomy.

In our study, 15 patients were examined for the presence of ANCA before and after proctocolectomy. Two patients initially positive for ANCA became negative and eight showed lower ANCA titres after proctocolectomy. Thus proctocolectomy decreased the ANCA activity significantly in most of our patients. The decline in ANCA titres after proctocolectomy suggests that by reducing inflammation or the available antigenic material proctocolectomy modifies the activity of the immune disturbance related to ulcerative colitis.

In most studies on ulcerative colitis, in which testing for specific ANCA associated antigens has been carried out, no significant reactivity with proteinase- 3 or myeloperoxidase has been detected. ${ }^{2518}$ In a recent study, Ellerbroek et al reported that $22 \%$ of ulcerative colitis patients positive for $\mathrm{p}$-ANCA recognised lactoferrin and $19.5 \%$ cathepsin G. However, p-ANCA occurring in inflammatory bowel disease still has an undefined major target antigen. ${ }^{19}$ This is in accordance with our study, as we tested all patients' sera both pre- and postoperatively by enzyme immunoassay for myeloperoxidase and proteinase- 3 antibodies and found that all patients were myeloperoxidase antibody negative and only one patient was proteinase- 3 antibody positive. While it is unusual to see proteinase- 3 antibodies manifest as p-ANCA, it remains possible that a non-specific antibody reaction was causing even this finding. In fact, serum antibodies to dietary agents such as cow's milk proteins that are widely used as blocking agents in enzyme immunoassays are not unusual in ulcerative colitis. ${ }^{20}$

Duerr $e t a l^{1}$ suggested that the presence or absence of ANCA could be used to distinguish between primary sclerosing cholangitis and other chronic hepatobiliary inflammatory diseases. However, p-ANCA has been reported to occur with other hepatobiliary diseases, for example autoimmune hepatitis and non-A, non-B, non-C hepatitis, although the prevalence is lower than that in primary sclerosing cholangitis. ${ }^{7}$ No correlation between ANCA and histological stage of primary sclerosing cholangitis or liver function values in patients with this condition has been found..$^{921} 22$ In our study, all three patients with fibrous cholangitis (one with primary sclerosing cholangitis proven by cholangiography, two with liver histology consistent with small duct primary sclerosing cholangitis) and all patients with non-specific reactive hepatitis at liver biopsy at proctocolectomy were positive for p-ANCA. However, ANCA were also detected among patients with normal liver histology.

Our study supports earlier findings in showing a high prevalence of ANCA in ulcerative colitis. After proctocolectomy the ANCA titres decreased in the majority of our patients, suggesting that reduction of the inflammation or the available antigenic material modifies the immune disturbance related to ulcerative colitis. Whether ANCA can be used to indicate the disease activity of ulcerative colitis in relation to therapeutic measures other than proctocolectomy requires further study.

1 Jennette JC, Falk RJ. Antineutrophil cytoplasmic autoantibodies and associated diseases: a review. $A m \mathcal{F}$ Kidney Dis 1990;15:517-29.

2 Saxon A, Shanahan F, Landers C, Ganz T, Targan S. A distinct subset of antineutrophil cytoplasmic antibodies is associated with inflammatory bowel disease. $\mathcal{f}$ Allergy Clin Immunol 1990;86:202-10.

3 Duerr RH, Targan SR, Landers CJ, Sutherland LR, Shanahan F. Anti-neutrophil cytoplasmic antibodies in ulcerative colitis. Gastroenterology 1991;100:1590-6.

4 Cohen Tervaert JW, Huitema MG, Hene RJ, Sluiter WJ, The TH, Van der Hem GK, et al. Relapses of Wegener's granulomatosis: prevention by treatment based on antineutrophil cytoplasm antibody levels. Lancet 1990;336: 709-11.

5 Cambridge G, Rampton DS, Stevens TRJ, McCarthy DA, Kamm M, Leaker B. Anti-neutrophil antibodies in inflammatory bowel disease: prevalence and diagnostic role. Gut 1992;33:668-74.

6 Oudkerk Pool M, Ellerbroek PM, Ridwan BU, Goldschmeding R, von Blomberg BME, Pena AS, et al. Serum antineutrophil cytoplasmic autoantibodies in inflammatory bowel disease are mainly associated with ulcerative colitis. A correlation study between perinuclear antineutrophil cytoplasmic auto-antibodies and clinical antimeters, medical, and surgical treatment. Gut 1993;34: meters,

7 Hardarson S, LaBrecque DR, Mitros FA, Neil GA, Goeken JA. Antineutrophil cytoplasmic antibody in inflammatory bowel and hepatobiliary diseases. Am f Clin Pathol 1993 99:277-81.

8 Olsson R, Danielsson Å, Järnerot G, Lindström E, Lööf L, Rolny $\mathrm{P}$, et al. Prevalence of primary sclerosing cholangitis in patients with ulcerative colitis. Gastroenterology 1991; 100:1319-23.

9 Seibold F, Weber P, Klein R, Berg PA, Wiedmann KH. Clinical significance of antibodies against neutrophils in patients with inflammatory bowel disease and primary sclerosing cholangitis. Gut 1992;33:657-62.

10 Reumaux D, Colombel JF, Duclos B, Chaussade S, Belaiche $\mathrm{J}$, Jacquot $\mathrm{S}$, et al. Anti-neutrophil cytoplasmic auto-antibodies in sera from patients with ulcerative colitis after proctocolectomy with ileo-anal anastomosis. In: Gross proctocolectomy with ileo-anal anastomosis. In: Gross WL, ed. ANCA-associated vasculitides: immunol
clinical aspects. London: Plenum Press, 523-6.

11 Ludwig J, Barham SS, LaRusso NF, Elveback LR, Wiesner RH, McCall JT. Morphologic features of chronic hepatitis associated with primary sclerosing cholangitis and chronic ulcerative colitis. Hepatology 1981;1:632-40.

12 Ludwig J, LaRusso F, Wiesner RH. Primary sclerosing cholangitis In: Peters LS, Craig R, eds. Liver pathology. New York: Churchill Livingstone, 1986:193-219.

13 van der Woude FJ, Rasmussen N, Lobatto S, Wiik A, Permin $\mathrm{H}$, van Es LA, et al. Autoantibodies against neutrophils activity in Wegener's granulomatosis. Lancet 1985; i:425-9.

14 Mustonen J, Soppi E, Pasternack A, Hällström O. Clinical significance of autoantibodies against neutrophil cytoplasmic components in patients with renal disease. $A m \mathcal{F}$ Nephrol 1990;10:482-8.

15 Lock RJ. Detection of autoantibodies to neutrophil cytoplasmic antigens. F Clin Pathol 1994;47:4-8.

16 Goeken JA. ANCA in inflammatory bowel and liver disease: new evidence for an autoimmune etiology? Clin Immunol 1993;13:119-25.

17 Rump JA, Wörner I, Roth M, Schölmerich J, Hänsch $M$, Peter HH. P-ANCA of undefined specificity in ulcerative colitis: correlation to disease activity and therapy. In: Gross WL, ed. ANCA-associated vasculitides: immunological and WL, ed. ANCA-associated vasculitides: immunological
clinical aspects. London: Plenum Press, 1993:507-13.

18 Rump JA, Schölmerich J, Gross V, Roth M, Helfesrieder R, Rautmann A, et al. A new type of perinuclear antineutrophil cytoplasmic antibody (p-ANCA) in active neutrophil cytoplasmic antibody (p-ANCA) in active
ulcerative colitis but not in Crohn's disease. Immunobiology ulcerative colitis but

19 Ellerbroek PM, Oudkerk Pool M, Ridwan BU, Dolman $\mathrm{KM}$, von Blomberg BME, von dem Borne AEG, et al. Neutrophil cytoplasmic antibodies (p-ANCA) in ulcerative colitis. $\mathcal{f}$ Clin Pathol 1994;47:257-62.

20 Aitola PT, Soppi ET, Halonen PJ, Laine ST, Matikainen MJ. The effect of proctocolectomy on serum antibody levels against cow's milk proteins in patients with chronic ulcerative colitis, with special reference to liver changes. Scand $\mathcal{F}$ Gastroenterol 1994;29:646-50.

21 Duerr RH, Targan SR, Landers CJ, LaRusso NF, Lindsay KI, Wiesner RH, et al. Neutrophil cytoplasmic antibodies: a link between primary sclerosing cholangitis and ulcera link between primary sclerosing cholangitis and

22 Lo SK, Fleming KA, Chapman RW. Prevalence of antineutrophil antibody in primary sclerosing cholangitis and ulcerative colitis using an alkaline phosphatase technique. Gut 1992;33: 1370-5. 